\title{
Trauma, violence and recovery in the life stories of people who have injected drugs
}

\author{
Richard Hammersley, ${ }^{1}$ Marie Reid, ${ }^{1}$ Phil Dalgarno, ${ }^{2}$ Jason Wallace, ${ }^{3}$ Dave Liddell ${ }^{3}$ \\ ${ }^{1}$ Department of Psychology, University of Hull; ${ }^{2}$ School of Health and Life Sciences, Glasgow Caledonian University; ${ }^{3}$ Scottish \\ Drugs Forum, Glasgow, UK
}

\begin{abstract}
This paper reports qualitative data about violence and trauma amongst drug injectors in Scotland, collected using a life story method with 55 drug injectors currently in recovery. It focusses on different types of violence and trauma that occurred whilst using drugs. Stories told of very severe and repeated traumas often involving extreme violence. One motive for escalating and continuing drug use was avoidant coping to deaden the emotions and thoughts regarding these traumas. Many traumas were due partly to the criminal drug milieu, so respondents were in a vicious cycle of using opiates and other sedative drugs to cope with the consequences of being involved in a lifestyle of using, supplying and obtaining illegal sedative drugs. Drugs were used as a dynamic method of self-medication or avoidant coping to cope with life in a criminal milieu. Traumatic events were more often described as worsening the lifestyle than as being motives for quitting. Amongst the traumas recounted were many incidents of extreme violence. Using drugs to block the psychological effects of such traumas may help explain why people persist using opiates despite experiencing evident serious harms. Interventions need to recognise this, be trauma-aware and appreciate that violence and trauma have severe adverse effects on drug injectors even when they are themselves hardened and violent.
\end{abstract}

\section{Introduction}

The relationships between a lifestyle involving problematic drug use and violence are well documented ${ }^{1}$ and complicated by many factors including the illegality of drugs and the development of drug use and offending in a common causal nexus. ${ }^{2}$ Much research and writing about drugs and violence focusses on their impact on in-

Correspondence: Richard Hammersley, Department of Psychology, University of Hull, Cottingham Road, Hull HU6 7RX, UK. Tel.: +44 7979303951 .

E-mail: r.hammersley@hull.ac.uk

Key words: Drug injecting; heroin; trauma; violence; recovery; self-medication.

Conflict of interest: The authors declare no potential conflict of interests.

Acknowledgements: We would like to thank five fieldworkers who collected data and three former staff of Scottish Drugs Forum who facilitated the research but did not contribute to this paper.

Received for publication: 1 May 2020.

Accepted for publication: 4 October 2020.

This work is licensed under a Creative Commons Attribution NonCommercial 4.0 License (CC BY-NC 4.0).

${ }^{\circ}$ Copyright: the Author(s), 2020

Licensee PAGEPress, Italy

Qualitative Research in Medicine \& Healthcare 2020; 4:65-74

doi:10.4081/qrmh.2020.9073 dividual victims and wider communities. In contrast, this paper is about the impact of violence and other trauma on the life stories of individual problem drug users. ${ }^{3}$ It follows on from a previous paper using the same life story data set to examine the impact of childhood trauma on the development of drug injecting. ${ }^{4}$ The research had set out to study life stories, not trauma, but that was a dominant theme. Sadly, participants' stories accepted childhood loss, abuse and trauma as normal and did not tell of proactive adult concern about either their losses, neglect and maltreatment or the, often obvious, effects on their behaviour. We also concluded that "Many of the difficult behaviours exhibited by addiction service clients may be due to trauma, rather than to drug effects, drug dependence, fecklessness, or being antisocial or aggressive by nature" (p13). Analysing the childhood data, we noticed that participants also told of considerable trauma whilst using drugs, especially from violent events. The present analysis aimed to explore this further, with the aim of considering how violence and trauma interact with problematic drug use. As will be explained, we used a theoretically derived scheme of themes. In Scotland, drug injecting has been an indicator of 'problem drug use' since the early 1980s, so here the target population was people who had injected drugs. This criterion was chosen because most Scottish drug injectors use multiple classes of controlled drugs, and drink alcohol as well, so focussing on heroin, or opiates in general is inappropriate.

There is a plenitude of research documenting violence and trauma amongst drug injectors, but only rarely is there discussion of causation beyond attributing violence to the nature of drugs, drug addicts and their illegal activities. 
Part of the stereotyping of drug injectors is to depict them as evil, unempathetic due to addiction and/or personality, and lacking volitional control of their actions. ${ }^{5}$ It is less often considered that they too suffer as both victims and perpetrators of violence..$^{6-8}$

Theory about drugs and violence tends towards theorising that either problem drug users are the victims of their circumstances and history, or that they have major psychological problems due to predisposition. Circumstance theories tend to assume that problem drug users are only involved in violence because they have no choice and that they suffer because of it, while predisposition theories tend to assume that they choose violence and are inured to it. In reality, the two types of explanation interact, and people can be both damaged victims and vicious perpetrators. A third type of explanation, intoxication, explains violence as directly caused by the acute effects of drugs or by the chronic effects of persistent use.

Two broad classes of circumstances have been proposed to explain drug related violence: i) Circumstances of enforcement, when buying and selling illegal drugs are policed by threats of, and actual, violence; ${ }^{910}$ ii) Cultural circumstances, when problem drug use (typically involving opiates, stimulants or both) occurs in a deviant subculture ${ }^{11}$ where certain forms of violence are considered normal, or instrumentally necessary. Within such a subculture, skill at violence and tolerance for it may facilitate success. ${ }^{7,8}$ Globally, drug supply and use often occur within gang culture, where the gang uses violence to enforce power in general rather than drugs trade specifically. ${ }^{12-16}$

Here, we will refer to milieu rather than subculture because the life story approach is about the individual story rather than about a shared cultural understanding such as might define a subculture. Most Scots who inject have injected heroin, but they usually also take and inject other drugs. ${ }^{17}$ For life stories involving drug injecting, the concept of a subculture also may be problematic because people in recovery often emphasise that the lifestyle of injecting heroin or other drugs is inauthentic and based only on a shared desire for drug use by people who otherwise have little in common..$^{18}$ However, ethnographic accounts of how social groups of drug injectors function suggest a subculture at the time, which can be durable and have positive attributes such as caring, sharing, compassion and mutual support. ${ }^{7,18}$ Here milieu is used to encapsulate these sociological complexities.

There are also two broad types of explanation of personal predispositions that facilitate violence: iii) Predisposed by trauma - as reviewed in Hammersley et al. ${ }^{4}$ many problem drug users have PTSD, which can lead to violent behaviour in some situations; iv) Predisposed by personality - some personality traits and developmental and acquired disorders, including brain damage, make people more easily capable of violence against others. Such dispositions are over-represented amongst problem drug users and prison inmates. ${ }^{1,2}$ Childhood trauma is often theorised to be a causal factor for adult psychological problems, ${ }^{19}$ so these explanations interact.

Finally, v) Intoxication facilitates violence by impairing multiple cognitive processes affecting mood, notably irritability, decision-making and forward planning, the interpretation of interpersonal communication, impulse control and pain perception. Such effects are documented for alcohol, opiates, benzodiazepines and stimulants. ${ }^{2}$ Here, in a paper about life stories rather than neuroscience, we shall not separate out acute intoxication from longer-term drug effects, or effects of withdrawal.

A problem to be noted with research on drugs and violence is that in the literature the effects of alcohol overwhelms the effects of drugs. ${ }^{20,21}$ Moreover, not all studies report a detailed substance use history, so it is very difficult to connect violent behaviours to specific drug combinations. We shall go on to propose that, generally, a drug's neuropsychological effects causing violence is too simple an understanding anyway.

Here, using elicited life story data collected from problem drug users in recovery in Scotland, ${ }^{22}$ we document the often-extreme violence in the drug injecting milieu, propose a model of how problem drug use and violence develop together, and how the two interact to impede recovery. In brief, a vicious cycle tends to develop where sedative drugs are used to escape from the psychological consequences of violence and other trauma, with both being increasingly caused by the injecting and drug supply milieu, which the person is involved in mainly to obtain the sedative drugs that enable them to cope with the associated violence and trauma.

Previously, ${ }^{4}$ we documented the severity of trauma in the childhood stories of many people who are recovering from problem drug use in Scotland. They often told of violence and abuse that were much more protracted and severe than was normative in the culture they grew up in. Alcohol abuse by parental figures was often involved. Many of the cohort felt that their trauma was directly related to their subsequent drug problems. It could lead directly to precocious substance abuse as a form of avoidant coping, to forming relationships with friends or partners who were problem drug users, drug dealers, or criminals, and simply to heavy substance use which formed a basis for the subsequent development of a drug problem in late adolescence or early adulthood, which was often triggered by further trauma. A minority of the cohort described severe behavioural problems as children that led to their being placed in care and/or abused, then led to problem drug use.

This paper continues the lifespan narrative through adolescence to adulthood, focussing on trauma. Participants told of a wide variety of traumas and life challenges, but the high incidence of events involving violence was particularly striking to the researchers. Therefore, this analysis focusses on how violence whilst adult fit into drug users' life stories, structuring the results around the 
five causal themes outlined above: Trauma, Enforcement, Culture, Personality, Intoxication; then goes on to consider how trauma and violence affect recovery. The aim of this analysis is to elucidate the impact of violence on the lives of people who have injected drugs.

\section{Methods}

Dan McAdams' Life Story Method (see www.sesp. northwestern.edu/foley/, accessed 23/5/2012) was used to collect narrative data about the person's entire life, using a semi-structured interview. This asks participants to divide their lives into chapters (here TV episodes was the substitute metaphor) and describe them, then describe a series of more focussed events such as the worst thing that ever happened to you and the wisest thing you have ever done. It also asks specific questions about life.

Theoretical assumptions ${ }^{23}$ include that personal identity is constructed as a story, and that life stories have common, classifiable themes, characters and structures, which are informative about the person's identity. The life story method encourages systematic recall of key aspects of the person's life, which in turn allowed participants to place drug use as centrally or peripherally in their stories as they wished. This was deliberately to contrast with the normal tendency for research with drug users to focus on drug use, risk factors for drug use and harms caused by drug problems.

The research team that designed, implemented and analysed the research had multiple perspectives as it included academics with considerable experience of addiction research, staff of Scottish Drugs Forum (SDF), which is Scotland's main umbrella organisation for charitable or third sector work against drug problems, and SDF volunteers who served as peer research fieldworkers.

The original research intent was to strengthen the voices of drug users themselves within discourse about drug problems and provide information about the nature of their lives, which was why the life story method was chosen. The method was adapted by research stakeholders after discussion by simplifying the definition of different periods to childhood, adolescence, adulthood before injecting, adulthood with injecting, and afterwards.

Fieldworkers were SDF volunteers trained to conduct peer research, themselves recovering from substance use problems and free of illicit drug use. They had already been assessed and trained to work as volunteers within SDF. They were additionally assessed for this particular project and supported throughout it by their case workers and the SDF User Involvement Development Officer, who directly oversaw the fieldwork. Training comprised some 50-60 hours of general training from SDF staff, plus some nine hours of specific training in the interview method and rationale for the research. During training, the interview method was discussed and modified according to trainee fieldworker feedback. Sixteen peer researchers started the specific training process of whom six completed it and became fieldworkers. Feedback from the other 10 indicated that during training, especially the roleplay/ practice part, they did not feel confident to handle the interview process without detrimental effects on themselves. The six fieldworkers completed the research without reporting any such effects.

\section{Participants}

Participants had recently been heroin injectors, which does not mean that their drug use was limited to heroin or to injecting drugs. Drugs commonly used with opiates in Scotland 1980-2011 included benzodiazepines, alcohol, methadone and cannabis, while relatively few Scots had a primary problem with stimulants. Participants and fieldworkers were in recovery. Practices and philosophies of recovery are varied in Scotland. ${ }^{24}$ Practices include 12steps abstinence-based fellowships, recovery communities where people reduce their use of illicit substances and also substitute prescribing of opioids, usually methadone, but sometimes buprenorphine. Individuals ultimately decide themselves the appropriate role and duration of substitute prescribing; whether abstinence involves abstaining only from substances that caused problems, or also from others with the potential to cause problems and whether prescribed medication, especially substitute opiates, are exempt from abstinence; as well as the use of mutual assistance organisations (e.g. Narcotics Anonymous).

Eligible participants had injected drugs within the previous five years. All the achieved cohort were currently in recovery and had received some form of intervention for their drug dependence at some point. They were recruited through drug agencies, support and recovery networks, and personal contacts during April to July 2011. Deliberately, recruitment occurred in cities, in post-industrial towns and in predominantly rural areas. To ensure confidentiality, precise recruitment sites are withheld. Participants were given a $£ 15$ shopping voucher as a disturbance allowance. Interviews took place on the premises of diverse recovery organisations in Scotland.

Of 55 participants, there were 38 men and 17 women. The age range at time of interview was 21 to 49 . All but one had at least one sibling and the mode was two. The majority of participants had worked at some point, although only about a third mentioned working in a stable way for periods of years as part of their stories, and most had children. Twenty-three had lived most in a city and the rest had mainly lived in a smaller place.

Most recalled some degree of trauma, often very severe, in childhood. Over a third of participants mentioned that at least one of their parents had had an alcohol or drug problem when they were growing up. More women than men had lived with a partner who also used drugs and, for women, it was often a sexual partner who had introduced them to heroin injecting. 


\section{Data analysis}

Interviews were digitally recorded then transcribed by a company specialising in medical and research transcriptions and accustomed to transcribing Scottish accents. Transcripts were passed to the researchers for analysis, along with the recordings for reference. Recordings were deleted after editing the transcripts to improve meaning and anonymise all identifying information.

We analysed the data in this paper in three stages. First, we conducted a content analysis of each of the structured questions, classifying the answers, then if appropriate over-arching themes were extracted from the content in a top-down way. Second, a general thematic analysis extracted common themes from both the life story structured into episodes and the answers to the more structured questions, at this stage the overarching theme of trauma emerged. Third, following on from our previous paper, ${ }^{4}$ the data were re-examined by re-reading the coding from the second stage, then revisiting the original data, and coded for the five theoretically derived themes described above: Trauma, Enforcement, Intoxication, Culture, Personality. Themes are presented in this order because some extracts from the interviews illustrate more than one theme. Finally, the impact of trauma on recovery is discussed.

The first author led on data analysis, but the interleaved processes of analysis and write-up were recursive. At each of the three stages, initial drafts were read and discussed with the other academics and practitioners on the team, including those themselves in recovery, in order to synthesise their various academic or theoretical, practitioner and recovery perspectives.

\section{Results}

\section{Violence and trauma in life stories}

Within the life story interviews, stories of violence and trauma were told as parts of the narrative of the different episodes in people's lives. As will be seen below, violence and trauma could be formative aspects of the development of a drugs problem, and also of the development of a life of offending, including time in prison. Stories of violence and trauma also came up as answers to the specific questions posed about life including: The worst thing that happened; something that changed your life for the worse; vivid adult memory; most stupid thing you ever did; biggest life challenge; bereavement; greatest failure or regret.

The majority of the life stories included violence and trauma, but a minority did not. Of these, three stories contained relatively little detail of any kind. Another two of the youngest respondents, in their early twenties, had quickly got methadone prescriptions and left the drug milieu. Seven respondents' stories, more than the others, involved legitimate employment and less drug supply and crime. Finally, two respondents had spent most of their adult lives in prison. Although violence and trauma do occur in prison, this limited their possibilities for experiencing the kinds of trauma reported here.

\section{Trauma}

Some people told of already using drugs heavily, then converting to more problematic patterns of use after a specific major traumatic event. More problematic patterns of use including starting injecting, initiation into heroin use and going off the rails and no longer caring about managing work, family life or anything else. Specific traumatic events included the sudden death or murder of a family member, sometimes multiple losses in a short time period, breakup with a partner, and the sudden and unexpected loss of access to children. Although some of these traumatic events were related to drug use or dealing, others were not. Perhaps the most extreme example was a man whose infant son died of cot death then his mother, father and younger brother also all died within a very short space of time.

Nobody told of killing anyone else, but this would have violated the agreed limits of respondent-researcher confidentiality. However, some respondents described perpetrating acts of serious violence against other people including family members, usually for what were seen as good reasons at the time. There was also violence enforcing drug trade and other criminal activity. As will be seen in the quotes below, these different types of violence were not always tidily separated.

Respondents tended to take the death of family members badly, especially if there were multiple deaths and if the death had been sudden and violent. A commonplace assumption in addiction research is that people become inured to the violence of the lifestyle. In reality, even respondents who were themselves hardened perpetrators of violence took sudden violent bereavements badly. Exposure to firearm violence at least is clearly associated with PTSD. ${ }^{20}$ Firearms are rare in Scotland, but some of the cohort mentioned events involving guns. One man said:

I have had guns put at my head a couple of times. (...) I mean when that happened to me last time it happened it wasn't long before I came into treatment, and I told them to pull the trigger.

Did you?

Which is not a clever thing to do.

No [laughter].

But it was kind of my mate got smashed by a sledgehammer right in front of us, right beside us at the back of the motor, and I thought the two of us were getting it.

A woman told of being shot with a shotgun leaning 
out of a window as the worst thing that had ever happened to her:

A guy went to shoot my man and I got it, and I play the video sometimes that can come up and play that in my head sometimes, and the shock I felt with that. (...) I think that is the worst thing that ever happened.

The graphic detail of these and other quotes are reminiscent of the vivid memories that occur for traumatic events. Another man had gone to prison for shooting his sister's boyfriend:

Well I done eight years out of the twelve. I shot my wee sister's boyfriend during a stupid drunken fucking pill fuelled fucking anger rage thing. Anyway, I got the sentence, done eight years out of it (...) The Saturday previous to my liberation two boys kicked in my wee brother's front door, sprayed him with petrol, torched him. I got out on the Friday and he died on the Tuesday. Two weeks after they buried him, my mum died. My head went right up my arse.

Some participants also told of the non-violent losses of relationships and/ or access to their children, which had devastating effects on them and increased their drug use. One man who had been bringing up his daughter told about the following losses. He was already using drugs at the time, but not yet heroin, but he began in jail when his mother died.

So, I brought the wean up there along with my mum and then ended up getting the jail and shit, done a stint in the jail, lost my mum when I was in the jail. I was only out months... No, two weeks before I got out my dad couldn't cope with the wean, so the wean went back to the mum and then I was only out about two months and my dad died, and it's just, my head was up my arse then with losing my mum, the wean, then losing my dad. Even though he was a bastard and all that, he was still my dad, you know?

The reason he gave for starting heroin was:

To block everything out of my head more or less, but it blocked it out that night, but it was there the next day again, even worse.

Another man, who had told of an unusually delinquent adolescence started taking heroin when he came out of five years in prison for attempting to rob a post office, but lost access to his wife and child because she had started seeing someone else:
It was about that time I just started, I started taking kit [heroin]; I started injecting kit. I started smoking it in the [Clinic1], kipped at my mum's, started smoking it at the [Clinic1], I liked the feeling - it took everything away, you know the madness, not the madness, the emotions. It took the emotions away and it just numbed me up for a bit. I was out in homeless places...

His drug use got worse and he ended up seriously fighting with his brothers about drugs while he was drunk:

And I went up the stairs after robbing this guy, I had three ounces of kit right, I'd stashed them in the park and I took one up with me and I went in the toilet and had a hit out of it, and went up the stairs and realised I'd left it in the toilet. I went back down and he's pouring it down the toilet. Oh! So, I stuck the nut on [headbutted] him and he's fell in the bath and I've about turned and walked out the house.

His other brother then attacked him with a spade, then he then told them he was going to kill them, went home got a knife and attacked both brothers, stabbing one multiple times. He described himself as "mad with drink" and mentioned that this was also so when he tried to hold up the post office. He now saw all this as totally unjustifiable. He was put back in prison for this, having been charged with premeditated attempted murder.

On release, it came out that his grandfather had been sexually abusing multiple children in the family, including the participant. He took his revenge by forcing the grandfather to give him all his money amongst other things. Then, his grandfather was assaulted and murdered by two men who found out about him being a paedophile. Later on, the participant tried to kill himself by being run over by a train.

Another man, who had a university degree turned to drugs in his mid 30s after he had been in a motorbike accident and taking prescription painkillers, then his wife of eight years left him and took their four-year-old daughter:

...turned out she had been abused by her dad as a young lassie. Right from she was five or three or something right to she was fifteen. Sexually, physically abused and mentally abused as well, he had committed suicide and he had hung himself so she could not get answers.

[...] We had a wee lassie and she couldn't handle the fact that her wee lassie was suddenly in a position that it could happen to her, no evidence or no reason to think it but just because of her past she thought her mum didn't protect her wondering if she could protect her lassie. So, she just upped and offed and that shattered me and that was the end of me. 
Another man with a long history of crime and drug use described his reaction to the love of his life dying in her 30s:

See the day before Partnerl got buried I went and got a turn (hit of heroin) and that is how bad my drug problem was, and I needed to focus, and to help myself manoeuvre and things like that and motivate myself. And leaving it to the last minute before I got ready to go and see, the done thing would have been for me to stay in the room with Partner1 the night before she was going away. That didn't happen I went and got a turn instead.

These extracts illustrate the wide range of severe traumas that respondents told of. It is important to emphasise that we have used illustrative quotes, not selected the worst ones for dramatic effect. There were drug trade related traumas and other types of trauma, sometimes multiple types. Traumas could quite directly escalate drug use, the drug using milieu increased the experience of trauma, and drug use made it harder to cope with trauma because opiates blocked experiencing and dealing with the emotional effects, which is generally required to recover from trauma. The severity of respondents' traumas could be such that one man told of being asked to leave a psychotherapy group for addicts because his life story was too upsetting for the other group members to cope with.

\section{Enforcement}

Respondents also told of serious violence in the context of enforcing the illegal drug trade and also of maintaining respect and status within a criminal community. Generally, people were more matter-of-fact about these stories, even making them comic, than about those of other kinds of trauma, but to the researchers the stories seem extreme and they are probably illustrative extreme examples rather than routine occurrences. The event when the respondent told the man with the gun on him to pull the trigger is one example. Another example was a man who described being pinned to the door of a car by a knife through his hand. Another example was from a man who had long been involved in criminal gangs as well as using drugs, who was an enforcer in prison:

And I would be on the phone he'd say scald him, I have to put sugar and (boiling) water in big continental cups and throw them at this guy's face. I have got to do this because if I don't then I am discredited, my credibility is out the window.

Interviewer: That's terrible (this is noteworthy as a rare occasion when the interviewer spontaneously judged the story).

Moreover, people who had committed such acts of enforcement, rather than being victims of them, now found it distressing that they had done these things, even although they seemed reasonable or necessary at the time.

\section{Violence and Intoxication}

Two of the above quotes mention intoxication as a factor in violence, although in the cohort this was rarely only on opiates. Alcohol and benzodiazepines were more commonly mentioned as specific factors, or simply being intoxicated on an unspecified mixture of drugs. Respondents told of being violent themselves whilst intoxicated and of the violent acts of other people while intoxicated. To illustrate, one woman described the violence of the men that she associated with. This account first describes them being attacked whilst in the house taking drugs and drinking, then goes on to describe how violent one of the cousins was, again it appeared usually when intoxicated.

And the next thing we were sitting in my house and I just seen a lot of shadows, and my veranda door had a bit of glass in it that you could see so I was like that. And there was only me, boyfriend's cousin 2, boyfriend's cousin 1 and Person 2 lying on my couch sleeping because the Celtic Rangers game had been on that day, but people must have thought everybody was still in watching the game, or having a drink or something... [...]

And the next thing the two doors went in right, and there was about thirteen of them or something and they have done boyfriend's cousin1 right in. You can see right in there right, and see that round bit that was sliced right in half, and he was stabbed about twelve times everywhere, all over the place. Boyfriend's cousin2 face was opened right up like that and Person2's head was like a road map, he woke up like what is going on and getting battered about with a machete.

She went on to describe the violent nature of one of the men who was attacked:

Boyfriend's cousin1 was a bastard as in his hands were like shovels and he was a mental job, so he was violently violent do you know what I mean [laughter], he was a bastard. He would batter you with a machete but don't get me wrong with the flat end and the blunt end but it still shows fuck.

Talking with the peer interviewers, respondents were generally matter of fact about such violence, but some of them indicated that with hindsight they felt that they would not, or should not, have done these things expect that they were intoxicated at the time. Although, some were clear that the effects of intoxication were not really separate from the effects of the milieu, where violence was part of life. 
Some respondents said that they now, in recovery, regretted things that they had done whilst intoxicated. But none told stories of feeling remorse at the time and a few explicitly said that the now regrettable violence seemed normal, even appropriate, at the time.

\section{Culture: the milieu itself}

This leads on to the impact of culture. Many respondents recognised that the culture they grew up in was often violent. Violence being most often related to disciplining children and partners, to heavy drinking and to gang fighting which was common in some parts of Scotland when these people were young. They tended to accept these common types of violence as normative. $^{4}$

However, respondents emphasised that some forms of violence they had experienced as children were not normative. Common non-normative forms of violence were being "very strict" and using physical punishment on children (often when drunk) and being habitually rather than occasionally violent to partners. Nobody mentioned perpetuating these forms on others as adults, although given the limits on research confidentiality, they would not have done. Most violence described was depicted as being part of the illegal drugs market and it generally occurred as the result of drug debits (see above), or other retribution. Some women also described having been in abusive relationships, sometimes multiple ones. Most of these abusive relationships were with men who used drugs. For example, one woman who married age 18 into what turned out to be a drug dealing family recalled her first violent fight with her husband:

I was as much to blame as him I see that now but I remember one day Daughterl stood in her coat screaming blue murder screaming going on for I think the reason I went I'd like to go out in Areal in the morning early, I can't remember the reason now and I bought a pair of shoes a cheap pair of fucking shoes and there was murder and now it was how he was wanting money for drugs. A big fight started but I remember, I got on with it but I remember I ended up in a corner and he spat on my face and that stuck in my head all through the lowest of the low, meaning how bad I felt then.

She then described escalating her drug use and beginning injecting in this context and the following quote also illustrates the impact of bereavement and the use of opiates to cope:

I was taking quite a lot myself, speed was a big part of drugs for me I used it through even taking kit I'd be up and down on speed as well. But he got, my mother in law got murdered in '92 and things had dramatically changed. I was getting
DFS from the doctor, I no really to take in the ri-, I took some didn't really like the feeling of them and it went dead quick within weeks to him coming back [probably from prison] cut out [i.e. some heroin], me snorting it and within a week I was jagging.

Two other examples of being enmeshed in a violent and traumatic milieu:

And it was all crooked people that my dad brought into the house, every one of them was crooked I didn't know anybody that wasn't crooked. [...] I can remember sitting in the house one day and he had four guys in and every one of them had been done with murder.

...My sister died. She actually came away from the drugs started to drink though, and her boyfriend got beeped from my cousin to go somewhere. He was still injecting and done everything until he cooked it up he started doing, she has been in a bath he went away. She went and took it and because she hasn't used it for, I don't know how long, she has OD-ed, somebody has phoned him there was somebody else in the house.

The story goes on to describe how his sister's boyfriend had cancelled the ambulance from concern over police involvement.

\section{Personality}

A significant minority of respondents told of childhoods that they considered suggested that they had grown up with major difficulties or problems that as far as they knew preceded childhood trauma and definitely preceded drug use. As adults, they were not necessarily distinguishable from other respondents who had been traumatised. One man explicitly said that he thought that he had ADHD. Others described being very impulsive and delinquent as children despite not recalling significant trauma that might have caused such behaviour.

Additionally, the content and style of another man's story strongly suggested ADHD. It did not appear that this had been fully recognised by family or health professionals although he was labelled hyperactive as a child. He told of some very serious and potentially traumatising events, most of which could be attributed partly to impulsivity, risk-taking and extreme intoxication, although he did not seem to explicitly make this connection. Unusually, he had mostly paid for drugs by having well-paid jobs as a milkman (in his teens), on a fishing boat, then in the merchant navy. Potentially traumatic events included drug related bereavement, the death of his father, which led to him using drugs even more heavily than ever, him assaulting other men on at least two occasions, and at least two car crashes: 
I passed my test and I had taken speed the night before I can't remember and I mind gouching and I hit a bus at eighty mile an hour. I nearly killed everybody on the bus, the engine and I walked out the car and I dragging the bus driver out the bus, I was screaming at him. And I kind of felt that I was going to punch him, and I had a couple of people I had on my back and I have mind me and my dad arguing because I didn't have a good relationship with my dad.

The trigger for his most recent residential rehabilitation was him being seemingly drugged and sexually assaulted whilst on leave from working on a boat.

\section{Impact on recovery?}

Most respondents told stories that involved multiple events that were traumatic and/or violent. As the above tellings illustrate, such events could lead to more serious use, such as injecting, to temporarily increased drug use, or indeed to continued heavy use. However, there were no discernible orderly connections between traumatic events and quitting, or between traumatic events and succeeding in quitting without relapse. In the stories, quit attempts were sometimes associated with positive events like the birth of children and sometimes with negative events, often very serious mental or physical health problems that had hospitalised and incapacitated them, reducing, at least for a while, their choice about quitting. Similarly, prison could lead to quitting or it could make problems worse. Methadone was also often involved in recovery. Some people eventually just decided to quit or go into recovery. But events around the quit attempt did not seem to predict success in any clear way. In the stories, violence and trauma played a stronger and clearer part in maintaining the lifestyle than in motivating cessation.

\section{Discussion}

To us, the data suggest that the drug injecting milieu often involved violence, which had distressing psychological effects on victims, perpetrators and their family and friends, sometimes resulting in bereavement. Superficially, this is obvious, but we feel that research and interventions on drug injecting and addiction tend to trivialise this distress. Active users and people in recovery tend to wave or laugh off their distress, at least in public, or attribute it to the inauthentic and dysfunctional nature of the milieu. ${ }^{19,20}$ Service providers tend to focus on the drugs as the root cause of violence which, if anything should be a motive for abstinence or reduction in use, so implicitly distress is conceptualised as caused by drugs, not by the violence itself. For example, the World Health Organisation suggests that drugs and violence are related due to psychopharmacology, economic compulsion and systemic mechanisms. ${ }^{25}$ Academics tend to be concerned with causation, so focus tends to be on understanding those mechanisms, which the present analysis indeed supports. However, to us, an essential further mechanism is that violence and trauma tend to increase and perpetuate substance use.

Drug injectors often played all three roles of victim, perpetrator and family member or friend, and experienced multiple distress. Some stories told of perpetrating violence whilst intoxicated, and some respondents now wished that they had not done such things. But there was also violence and trauma when intoxication may not have been a factor. A more substantial motive for using opiates and other sedative drugs was to block out the psychological effects of violence. Often the violence is linked to the supply of the very drugs being used to block the effects of violence; a vicious cycle. To escape this cycle, a drug injector needs to be able to stop using sedative drugs and also to be able to cope with the unblocked thoughts and feelings that emerge un-sedated. To do so while the relevant drugs are illegal and stigmatised required most people to move away from the milieu geographically, or socially and psychologically.

Furthermore, taking an overview of these life stories, rather than being a drugs milieu, the milieu more seems to us to be markedly interpersonally harsh, rough, violent and murderous, with drug and alcohol use being secondary. This is a milieu where one's brother can be unexpectedly set on fire as you are being released from prison. Much of the violence emerged from drug supply, but this occurred in the broader context of criminality. Drug injectors usually had to be involved in this criminal milieu because involvement was necessary to obtain drugs and often to fund drug use. So, the milieu or setting of illegal drug use appeared to be an additional contributor to trauma, as well as individual circumstance and predisposition. We suggest that a vicious cycle tends to develop where sedative drugs are used to escape from the psychological consequences of violence and other trauma, with both being increasingly caused by the violent drug supply milieu, which the person is involved in mainly to obtain the sedative drugs that enable them to cope with the associated violence and trauma. We feel that there is complacency about this state of affairs, which would not be tolerated in any other sphere of mental health. It seems inconceivable, for example, that violence by and on people with intellectual difficulties nowadays would be dismissed as an inevitable consequence of their condition.

While drug supply continues to be illegal and entirely unregulated, it provides a bounteous commodity for criminals and opportunity for youth in areas where legal paid work is scarce.$^{26}$ Regulating drugs instead would weaken the links between their supply and violent criminal activity, and the stories here included many incidents of violence linked to drug dealing. However, drugs, especially sedative ones, had two other links to violence. Respon- 
dents were involved in some violence while they were intoxicated and often mentioned alcohol and benzodiazepines as specific factors. Second, much more importantly, it was clear from these data that the psychological aftermath of violence, whether as victim or perpetrator, was often managed by using drugs for sedation, to avoid thinking about the psychological trauma that the violence had caused.

The "self-medication" hypothesis has a long history in addiction research, ${ }^{27}$ and is often mentioned in clinical anecdotes, yet it has lacked convincing research support. ${ }^{28}$ Perhaps this is because it has usually been framed as selfmedication for a pre-existing psychiatric condition rather than being a dynamic method of coping with life in a criminal milieu. Recent findings indeed suggest that selfmedication is a dynamic feedback process where drug use can increase the likelihood of trauma. ${ }^{29,30}$ Here, indeed intoxication facilitated repeated involvement in traumatic acts, both as victim and perpetrator, that without self-medication might have become intolerable to the person.

Regarding whether heroin addiction is about self-medication to cope with childhood trauma, or about intoxication, ${ }^{31,32}$ these data suggest that both apply, plus self-medication - avoidant coping - to cope with trauma related to the milieu. Opiates and other sedatives are not specific medicines for trauma, but they are powerful tools to suppress cognitions and emotions that can be used to dull the pain of trauma. This use may also help answer the frequently asked question "Why do addicts persist with use despite very marked adverse consequences?" Perhaps this is because they learn to self-sedate with drugs to escape those adverse consequences leading to a vicious cycle that is difficult to break. This theory contrasts with predominant explanations of drugs themselves as the root explanation of self-destructive behaviour. For example:

\section{Brain imaging studies from people addicted to drugs show physical changes in areas of the brain that are critical for judgment, decision-making, learning, memory, and behavior control. Scientists believe that these changes alter the way the brain works and may help explain the compulsive and destructive behaviors of a person who becomes addicted. ${ }^{33}$}

For us, a danger of attributing violence and other dysfunctional behaviours to drugs' effects on the brain is that this minimises the need to understand and intervene regarding the appalling social and psychological conditions where drug injecting tends to occur. ${ }^{6}$ Additionally, trauma also has prolonged effects on brain structure and function $^{34}$ so, in the absence of relevant longitudinal imaging data, such changes may be reasons for substance abuse rather than consequences of it. Brain changes may reflect that a traumatised person may care less about themselves, be less concerned about life planning and be less inclined to avoid destructive behaviours, as told by participants here. Then of course chronic drug use may cause further neuropsychological changes.

Substitute prescribing of drugs is accepted to reduce offending and other problems related to the milieu, ${ }^{35}$ but it does not disrupt the violence and may even help perpetuate it by increasing the number of people who can remain sufficiently sedated to avoid dealing with their traumatic experiences. Substitute prescribing works best as an adjunct to a recovery that involves deliberately leaving the milieu and includes psychological intervention. Such interventions should be trauma aware, ${ }^{3,36}$ which extends to recognising that the perpetrators of violence can also be traumatised, and their traumatisation is often worsened by previous trauma. This applies even if they appear callous and lacking remorse, which are symptoms of PTSD.

Addiction services are working regularly with traumatised people and it is important for them to get the balance right between encouraging abstinence or reduced substance use and helping people overcome traumatisation, which is no less problematic for it being in some sense caused by drug use. Getting the balance wrong can have disastrous effects, ${ }^{6}$ as attempted abstinence or moderation without overcoming trauma may be unmanageable, but so may be overcoming trauma whilst chronically intoxicated.

It is likely that any sedating drug including alcohol can be used for avoidant coping, so further prohibitions on specific drugs may not be effective. Moreover, supply reduction could make the milieu more violent as prices, profits and risks increase. Reducing the violence associated with drug supply is the most logical way of breaking the vicious cycle. Much of that violence is instrumental in an industry that is illegal, has no recourse to the law and is completely unregulated, so regulation may be the best option for reducing drug-related violence, which in turn may facilitate recovery from opiate addiction. A wide variety of options for regulation exist, from supervised injecting, to the medical supply of some opiates (although see the concern about avoidant coping), to decriminalisation or legalisation of some drugs.

In summary, these data suggest that drug injecting is linked to violence in three ways: via instrumental violence in the drugs trade; violence whilst intoxicated; use of sedative drugs to cope with the adverse psychological consequences of violence. These create a vicious cycle of using drugs to cope with the terrible things that have happened due in part to being involved with illegal drugs. Often there are multiple traumas before a drug injector recovers and recovery needs to facilitate dealing with trauma as well as quitting drug use. Although competent addiction services already appreciate this and plan their interventions accordingly, there is a continued need for services to become more trauma aware and additionally to appreciate the cyclical relationship within the drug milieu between violence and sedative drug use. 


\section{References}

1. Duke AA, Smith KMZ, Oberleitner LMS, et al. Alcohol, drugs, and violence: a meta-meta-analysis. Psychol Violence 2018;8:238-49.

2. Boles SM, Miotto K. Substance abuse and violence: a review of the literature. Aggress Violent Behav 2003;8:155.

3. Rosenberg L. Addressing Trauma in Mental Health and Substance Use Treatment. J Behav Health Serv Res 2011; 38:428-31.

4. Hammersley R, Dalgarno P, McCollum S, Reid M, Strike Y, Smith A, et al. Trauma in the childhood stories of people who have injected drugs. Addict Res Theor 2016;24:135-51.

5. Hammersley R, Reid M. Why the pervasive addiction myth is still believed?. Addict Res Theor 2002;10:7-30.

6. Agar M. Ripping and Running: A Formal Ethnography of Urban Heroin Addictions. Seminar Press; 1973.

7. Bourgois P, Schonberg J. Righteous Dopefiend. Berkeley: University of California Press; 2009.

8. Levy JA, Anderson T. The drug career of the older injector. Addict Res Theor 2005;13:245-58.

9. Bender K, Thompson SJ, Ferguson K, et al. Substance use and victimization: Street-involved youths' perspectives and service implications. Child Youth Serv Rev 2012;34:2392-9.

10. Zweig JM, Yahner J, Rossman SB. Does Recent Physical and Sexual Victimization Affect Further Substance Use for Adult Drug-Involved Offenders? J Interpers Violence 2012;27:2348-72.

11. Seffrin PM, Domahidi BI. The Drugs-Violence Nexus: A Systematic Comparison of Adolescent Drug Dealers and Drug Users. J Drug Iss 2014;44:394-413.

12. Jacques S, Wright R, Allen A. Drug dealers, retaliation, and deterrence. Int J Drug Policy 2014;25:656-62.

13. Golub A, Johnson BD, Dunlap E. Subcultural evolution and illicit drug use. Addict Res Theor 2005;13:217-29.

14. Beynon CM, McVeigh C, McVeigh J, Leavey C, Bellis MA. The involvement of drugs and alcohol in drug-facilitated sexual assault: A systematic review of the evidence. Trauma Violence Abuse 2008;9:178-88.

15. Curtis R. The improbable transformation of inner-city neighborhoods: Crime, violence, drugs, and youth in the 1990s. J Crim Law Criminol 1998;88:1233-76.

16. Inciardi JA, Horowitz R, Pottieger AE. Street Kids, Street Drugs, Street Crime: An Examination of Drug Use and Serious Delinquency in Miami. J Contemp Crim Just 1992;8:365-7.

17. Hammersley R, Dalgarno P. Drugs: Policy and Practice in Health and Social Care. Edinburgh: Dunedin Academic Press; 2012.

18. Mullen K, Hammersley R. Attempted cessation of heroin use among men approaching mid-life. Drugs-Educ Prevent Policy 2006;13:77-92.

19. Carr CP, Severi Martins CM, Stingel AM, Lemgruber VB, Juruena MF. The Role of Early Life Stress in Adult Psychiatric Disorders: a Systematic Review According to Childhood Trauma Subtypes. J Nerv Ment Dis 2013;201:1007-20.

20. Montgomerie JZ, Lawrence AE, LaMotte AD, Taft CT. The link between posttraumatic stress disorder and firearm violence: A review. Aggress Violent Behav 2015;21:39.
21. Choenni V, Hammink A, van de Mheen D. Association Between Substance Use and the Perpetration of Family Violence in Industrialized Countries: A Systematic Review. Trauma Violence Abuse 2017;18:37-50.

22. Hammersley R, Dalgarno P. Trauma and recovery amongst people who have injected drugs within the past five years. Scottish Drugs Forum 2012. Available from: http://www. sdf.org.uk/wp-content/uploads/2016/08/Trauma_Recovery_Report.pdf

23. McAdams DP. The psychology of life stories. Rev Gen Psychol 2001;5:100-22.

24. Scottish Recovery Network. What is recovery? Scottish Recovery Network, 2014. Available from: https:/www.scottishrecovery.net/what-is-recovery/

25. Atkinson A, Anderson Z, Hughes K, et al. Interpersonal violence and illicit drugs. Liverpool John Moores University Centre for Public Health, 2009. Available from: https://www.who.int/violenceprevention/interpersonal_violence_and_illicit_drug_use.pdf. Accessed: September 2020.

26. Bourgois P, Kain Hart L, Karandinos G, Montero F. Coming of age in the concrete killing fields of the US inner city. In: MacClancy J, (ed). Exotic no more: anthropology for the contemporary world. 2nd ed. Chicago: University of Chicago Press; 2019.

27. Khantzian EJ. Addiction as a self-regulation disorder and the role of self-medication. Addict 2013;108:668-9.

28. Hall DH, Queener JE. Self-medication hypothesis of substance use: Testing Khantzian's updated theory. J Psychoactive Drugs 2007;39:151-8.

29. Garland EL, Pettus-Davis C, Howard MO. Self-medication among traumatized youth: structural equation modeling of pathways between trauma history, substance misuse, and psychological distress. J Behav Med 2013;36:175-85.

30. Lazareck S, Robinson JA, Crum RM, et al. A longitudinal investigation of the role of self-medication in the development of comorbid mood and drug use disorders: findings from the National Epidemiologic Survey on Alcohol and Related Conditions (NESARC). J Clin Psychiatry 2012;73:e588-93.

31. Lembke A. From self-medication to intoxication: time for a paradigm shift. Addict 2013;108:670-1.

32. Darke S. Pathways to heroin dependence: time to re-appraise self-medication. Addict 2013;108:659-67.

33. National Institute on Drug Abuse. The science of drug use and addiction: The basics, 2018. Available from: https://www.drugabuse.gov/publications/media-guide/science-drug-use-addiction-basics. Accessed: September 2019.

34. Yu M, Linn KA, Shinohara RT, et al. Childhood trauma history is linked to abnormal brain connectivity in major depression. Proc Natl Acad Sci USA 2019 National Academy of Sciences;116(17):8582-8590.

35. Independent Expert Working Group on Drug Misuse and Dependence. Drug misuse and dependence. UK guidelines on clinical management. Department of Health 2017. Available from: https://assets.publishing.service.gov.uk/government/uploads/system/uploads/attachment_data/file/673978/ clinical_guidelines_2017.pdf

36. Dass-Brailsford P, Myrick AC. Psychological Trauma and Substance Abuse: The Need for an Integrated Approach. Trauma Violence Abuse 2010 2010;11:202-13. 